Tamara de Souza Pereira ${ }^{1}$

Rafaela Corrêa Pereira ${ }^{2}$

Michel Cardoso de Angelis-Pereira ${ }^{1}$

${ }^{1}$ Departamento de Nutrição, Universidade Federal de Lavras (UFLA). Campus UFLA/DCA, Campus Universitário. 37200-000 Lavras MG Brasil.tamaraspereira@ hotmail.com

${ }^{2}$ Departamento de Ciência dos Alimentos, UFLA. Lavras MG Brasil.

\section{Influência de intervenções educativas no conhecimento sobre alimentação e nutrição de adolescentes de uma escola pública}

\author{
Influence of educational interventions on knowledge \\ about food and nutrition of adolescents in a public school
}

Abstract Eating habits of adolescents have been marked by high consumption of foods rich in fat, sodium and simple sugars which, together with a sedentary lifestyle, are directly related to the incidence of obesity and other chronic non-communicable diseases both among adolescents and in adulthood. This scenario highlights the urgent need to adopt food and nutrition education (FNE) programs. Thus, the scope of this study was to identify the eating habits and subsequently evaluate the degree of knowledge about food and nutrition of adolescents, intervene with EAN actions, applying two separate educational resources (lecture and quiz-type game) and compare the effectiveness of both in the educational process. The study included 59 adolescents between 13 and 16 years of age, enrolled in two classes of the eighth grade of a public school in the city of Lavras in the State of Minas Gerais. Results revealed the prevalence of inadequate dietary patterns marked by the high consumption of foods rich in simple sugars and fats. Both the application of the game and the lecture were effective in increasing the level of knowledge among adolescents and, when compared, there was no significant difference methods between them.

Key words Educational resources, Eating habits, Food and nutrition education, Adolescents
Resumo Os hábitos alimentares de adolescentes têm sido marcados pelo alto consumo de alimentos ricos em gorduras, sódio e açúcares simples que, somados ao sedentarismo, estão diretamente relacionados com a incidência de obesidade entre outras doenças crônicas não transmissíveis tanto nesta faixa etária como na vida adulta. Este cenário reforça a urgência de se adotar programas de educação alimentar e nutricional (EAN). Sabendo-se disso, o objetivo deste trabalho foi identificar o hábito alimentar e sequencialmente avaliar o grau de conhecimento sobre alimentação e nutrição de adolescentes, intervir com EAN, aplicando dois recursos pedagógicos distintos (palestra e jogo do tipo quiz) e comparar a eficácia de ambos no processo educativo. Participaram do estudo 59 adolescentes entre 13 e 16 anos, matriculados em duas turmas da oitava série de uma escola municipal do município de Lavras - Minas Gerais. Os resultados indicaram prevalência de padrão alimentar inadequado evidenciando o consumo elevado de alimentos ricos em gorduras e açúcares simples. Tanto a aplicação do jogo como a palestra se mostraram eficientes para aumentar o grau de conhecimento dos adolescentes e, quando comparados, os métodos não mostraram diferença significativa entre si.

Palavras-chave Recursos pedagógicos, Hábitos alimentares, Educação alimentar e nutricional, Adolescentes 


\section{Introdução}

Trabalhar com adolescentes é sempre uma tarefa desafiadora, sendo ainda mais instigante quando se pretende trabalhar a educação alimentar e nutricional (EAN) nessa fase de vida. A adolescência, que de acordo com a World Health Organization $(\mathrm{WHO})^{1}$, compreende a faixa etária entre 10 e 19 anos de idade, é um período caracterizado por profundas alterações físicas e comportamentais. Durante essa etapa vários fatores podem influenciar nas escolhas e nos hábitos que formarão a identidade destes indivíduos, distanciando-se dos comportamentos típicos da infância e adquirindo características e competências para a vida adulta.

Sabe-se, no entanto, que os hábitos alimentares desta faixa etária têm sido marcados pelo alto consumo de alimentos processados, como lanches fast food, refrigerantes e doces, ricos em gorduras, sódio e açúcares simples que, somados ao sedentarismo e ao longo período destinado à $\mathrm{TV}$, computador e videogames, estão diretamente relacionados com a incidência de obesidade entre outras doenças crônicas não transmissíveis durante a adolescência e a vida adulta ${ }^{2}$.

Segundo Yang et al. ${ }^{3}$, os hábitos alimentares inadequados e o alto índice de sobrepeso e obesidade entre os adolescentes já se apresentam como um grave problema de saúde pública mundial, o que reforça a urgência de se adotar programas de EAN tendo como público alvo principal estes indivíduos, destacando-se neste caso a escola como um dos canais mais efetivos para a incorporação destas ações.

Desta forma, as práticas de EAN devem ressaltar a importância do papel da alimentação saudável sobre a qualidade de vida dos indivíduos e, acima de tudo, possibilitar a formação de sujeitos autônomos em suas escolhas alimentares para que os benefícios sejam observados a longo prazo. Para tanto, a adoção de práticas construtivistas e intuitivas neste processo se destacam por tornar possível para os indivíduos, atribuir significado particular ao mundo real de acordo com seus processos mentais, sendo o conhecimento efetivado pela sua interação com o meio, levando em consideração aspectos sensoriais, sentimentais e intelectuais ${ }^{4,5}$.

Neste sentido, diversos métodos e recursos pedagógicos têm sido propostos para promover a EAN, sendo os mais efetivos aqueles que se utilizam do lúdico, do dinamismo e da socialização em detrimento de práticas puramente expositivas, com conteúdos desconexos da realidade do educando. Além disso, práticas que adotam recursos pedagógicos capazes de instigar o interesse para aprendizagem e que influenciam no comportamento alimentar e nutricional dos adolescentes, despertando neles a visão crítica sobre o tema, também têm se destacado pela eficácia ${ }^{6,7}$.

Porém, mesmo levando em consideração estes aspectos, muitas intervenções relatadas na literatura ainda se mostraram pouco efetivas ${ }^{8}$, reforçando a necessidade de se conduzir mais estudos que proponham entender como essas variáveis influenciam na construção do conhecimento dos educandos. Este fato é confirmado por Shaya et al. ${ }^{9}$, que após extensa revisão de literatura sobre métodos de intervenção nutricional com crianças e adolescentes, constataram que mesmo com a variabilidade de metodologias empregadas no que diz respeito aos recursos adotados, duração das intervenções, forma de obtenção e avaliação dos dados, os resultados se mostraram pouco eficazes e persistentes, sendo o entendimento do efeito dessas variáveis sobre a eficácia das ações propostas ainda limitado.

Sendo assim, o objetivo deste trabalho foi avaliar o hábito alimentar e, sequencialmente, o grau de conhecimento sobre alimentação e nutrição de adolescentes do ensino fundamental de uma escola pública do município de Lavras, Minas Gerais; intervir com EAN, aplicando dois recursos pedagógicos distintos (palestra e jogo do tipo quiz); e comparar a eficácia de ambos no processo educativo dos adolescentes, de forma a compreender como o recurso adotado influencia nas percepções e no nível de conhecimento dos participantes.

\section{Metodologia}

\section{Amostra}

O estudo foi realizado durante o segundo semestre de 2013 com 59 adolescentes, na faixa etária entre treze e dezesseis anos, matriculados em duas turmas da oitava série de uma escola municipal do município de Lavras - Minas Gerais. O estudo foi aprovado pelo Comitê de Ética em Pesquisa com Seres Humanos da Universidade Federal de Lavras (UFLA).

\section{Delineamento experimental}

A intervenção proposta neste estudo foi composta de quatro etapas, conforme apresentado na Figura 1.

Inicialmente, todos os adolescentes foram avaliados, individualmente, quanto aos hábitos 


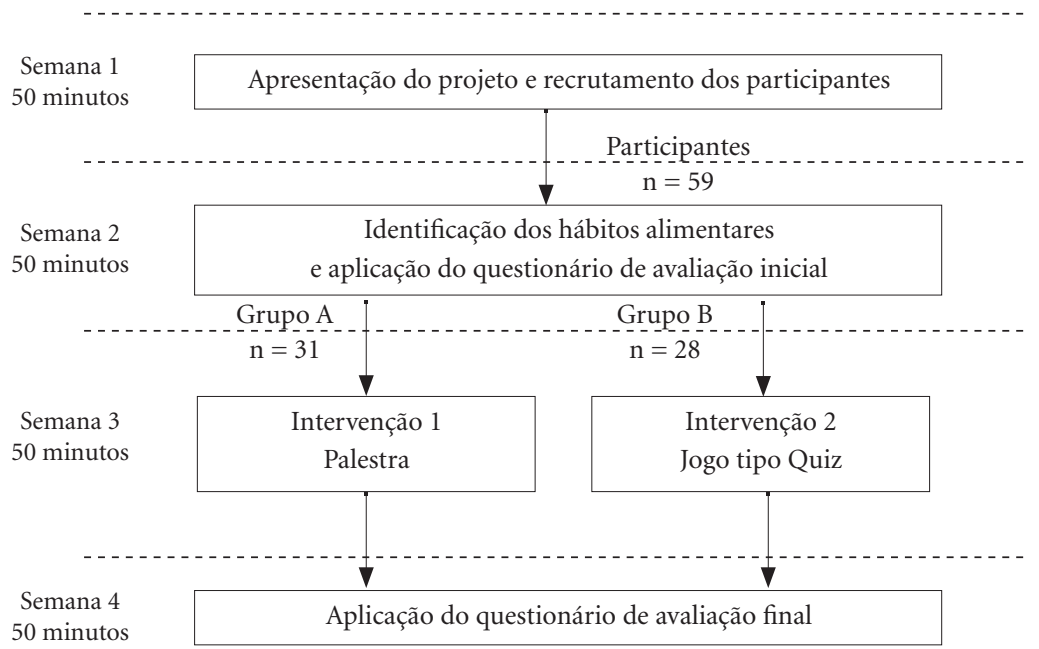

Figura 1. Fluxograma das atividades de intervenção nutricional conduzidas com os adolescentes de uma escola pública do município de Lavras - MG, Brasil, 2013.

de consumo de determinadas classes de alimentos (frutas, verduras e legumes, lanches fast food, doces e refrigerantes), quando foram orientados e acompanhados para fazer o preenchimento dos questionários de frequência de consumo, sendo o questionário elaborado de forma discriminada e com linguajar adequado para a idade. Em sequência, os adolescentes responderam um questionário estruturado composto por 30 questões referentes à nutrição e à alimentação saudável, abrangendo temas como os conceitos de nutrição como ciência e o papel do nutricionista, a importância da alimentação saudável e a classificação e a função dos nutrientes.

Sabendo-se das diferenças envolvidas entre a construção do conhecimento utilizando-se métodos tradicionais de ensino, como a palestra, em comparação com recursos dinâmicos, interativos e em equipe como os jogos, os adolescentes recrutados foram divididos em dois grupos (A e B), de acordo com a turma em que se encontravam matriculados. Ambas as turmas possuíam equivalência de aprendizagem e comportamento. Baseado nesta divisão, duas estratégias de intervenção em EAN foram então conduzidas com os adolescentes.

A primeira intervenção foi desenvolvida com o Grupo A por meio de palestra, abordando os seguintes temas: alimentação saudável; tipos, funções e fontes dos nutrientes; grupos alimentares, suas funções e número de porções recomendadas com base na pirâmide alimentar; a importância da água e a influência dos meios de comunicação na escolha dos alimentos.

A segunda intervenção foi desenvolvida com o Grupo B por meio de jogo do tipo quiz (jogo de perguntas e respostas), abordando os mesmo temas da intervenção realizada no Grupo A. Nesta atividade, o grupo foi dividido em duas equipes que respondiam alternadamente às perguntas de cada rodada. Após cada pergunta, seguiu-se uma explicação sobre o assunto abordado.

Uma semana após a aplicação das intervenções educativas, os adolescentes foram reavaliados por meio da aplicação de questionário, considerando os mesmos parâmetros do início da pesquisa, para se conhecer os resultados da intervenção e analisar as eventuais mudanças ocorridas. Todas as atividades tiveram duração de 50 minutos e foram conduzidas nas salas de aula da escola.

\section{Análises estatísticas e interpretação dos resultados}

Os dados obtidos no estudo foram tabulados e analisados por meio de parâmetros estatísticos descritivos e ANOVA, sendo as diferenças signi- 
ficativas entre os fatores em estudo avaliadas por Teste de Tukey ao nível de 5\% de significância. Utilizou-se nestas análises o software SPSS v. 20.0. Os dados foram submetidos ainda à análise de componentes principais (PCA), utilizando-se o software Chemoface versão $1.4^{10}$.

\section{Resultados e discussão}

Participaram do estudo 59 adolescentes, sendo $61 \%$ do sexo masculino e $39 \%$ do sexo feminino. Estes foram divididos em dois grupos, A e B, com 31 e 28 alunos, respectivamente. A faixa etária dos adolescentes participantes do estudo foi de 13 a 16 anos, sendo a média de idade de $14 \pm 1,29$ anos.

Os hábitos alimentares dos adolescentes que participaram da pesquisa revelou elevado consumo de alimentos altamente calóricos, ricos em açúcares simples, sódio e gordura (doces, lanches tipo fast food e refrigerantes) e baixo consumo de frutas, verduras e legumes, conforme apresentado na Tabela 1 .

Todos os estudantes relataram ter o hábito de consumir doces e guloseimas, sendo que $40,68 \%$ deles afirmaram consumir três ou mais vezes por dia. Os lanches tipo fast food também estiveram presentes na dieta de todos os estudantes, sendo que $16,95 \%$ relataram consumir esse tipo de alimento três ou mais vezes por dia. $\mathrm{O}$ consumo de refrigerantes pelo menos duas vezes ao dia foi relatado por $35,59 \%$ dos adolescentes. Em contrapartida, apenas $23,73 \%$ dos adolescentes relataram consumir frutas três ou mais vezes ao dia e $13,56 \%$ consumiam verduras e legumes com a mesma frequência. Considerando as recomendações da pirâmide alimentar adaptada para a população brasileira ${ }^{11}$ e o guia alimentar para a mesma $^{12}$, observa-se como esses dados são alar- mantes por não corresponderem às orientações desses guias, constatando o grande consumo de açúcares de adição, sódio e gorduras por meio de alimentos processados e ultraprocessados em detrimento do consumo dos alimentos ricos em vitaminas, minerais, demais substâncias com atividades antioxidantes e fibras como as frutas e demais vegetais in natura ou minimamente processados. Estes resultados corroboram vários outros estudos que constataram esse comportamento entre adolescentes ${ }^{13-18}$, evidenciando a urgente necessidade de intervenções educativas, principalmente por programas de políticas públicas para promover a adoção de práticas alimentares mais saudáveis.

Soma-se a este cenário, as percepções dos adolescentes em relação à alimentação e nutrição. Conforme apresentado na Figura 2, 96,61\% dos estudantes afirmaram saber o que é nutrição e quase a metade do grupo se preocupava com a alimentação (44,07\%); embora, 72,88\% nunca tenham feito uma consulta com nutricionista. Fica evidente, portanto, que estas áreas do conhecimento, apesar de não serem completamente desconhecidas, são marcadas pela relação limitada entre estes indivíduos e os profissionais da área. Além disso, nota-se que apenas 10,17\% dos adolescentes tinham o hábito de ler os rótulos dos alimentos e $28,81 \%$ deles achavam que a mídia exerce alguma influência sobre suas escolhas alimentares, o que é outro fator preocupante, além de indicar o desprovimento de EAN sobre eles, uma realidade que acomete a maior parte das regiões do Brasil. Este cenário é constatado por Freitas et al. ${ }^{19}$ em estudo que analisou as políticas públicas de nutrição para o controle da obesidade em adolescentes. Os autores afirmam que, no Brasil, essas políticas têm atuado de forma incipiente quando destinadas ao adolescente no que diz respeito ao fator obesidade. No entan-

Tabela 1. Consumo alimentar de adolescentes de 13 a 16 anos de idade $(n=59)$ de uma escola pública do município de Lavras - MG, Brasil.

\begin{tabular}{lrrrrr}
\hline \multirow{2}{*}{ Frequência de consumo } & Frutas & Verduras e legumes & Lanches (fast food) & Doces & Refrigerantes \\
\cline { 2 - 5 } & \multicolumn{5}{c}{$\mathbf{n}(\%)$} \\
\hline 1 vez/dia & $20,34(12)$ & $22,03(13)$ & $8,47(5)$ & $13,56(8)$ & $11,86(7)$ \\
2 vezes/dia & $16,95(10)$ & $42,37(25)$ & $8,47(5)$ & $20,34(12)$ & $35,59(21)$ \\
3 ou mais vezes/dia & $23,73(14)$ & $13,56(8)$ & $16,95(10)$ & $40,68(24)$ & $22,03(13)$ \\
1 a 2 vezes/semana & $35,59(21)$ & $13,56(8)$ & $66,10(39)$ & $25,42(15)$ & $28,81(17)$ \\
Nunca & $3,39(2)$ & $8,47(5)$ & $0(0)$ & $0(0)$ & $1,69(1)$ \\
Total & $100(59)$ & $100(59)$ & $100(59)$ & $100(59)$ & $100(59)$ \\
\hline
\end{tabular}




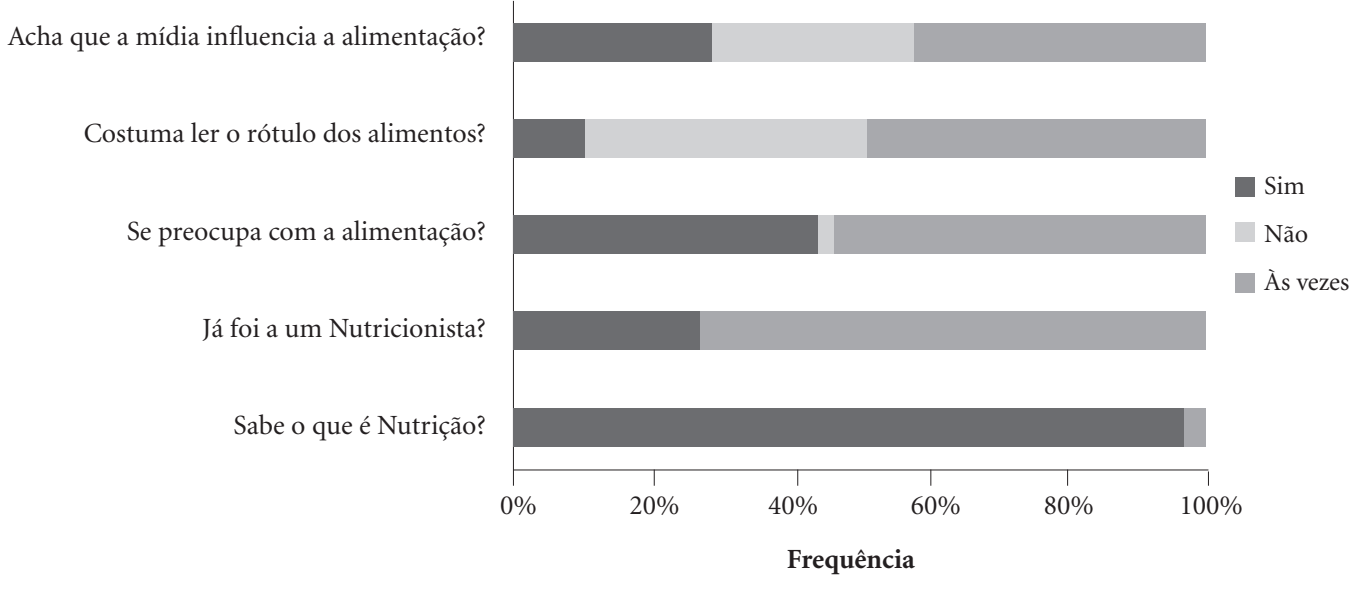

Figura 2. Comportamento dos adolescentes em relação à alimentação e nutrição.

to, segundo Wong e Carvalho ${ }^{20}$, a necessária implementação dessas políticas e a regulamentação das leis ainda estão longe de serem feitas.

Santos et al. ${ }^{21}$, objetivando analisar a quantidade e o horário das propagandas veiculadas pela televisão sobre produtos alimentícios e classificá-los de acordo com a pirâmide alimentar, identificaram que das 239 propagandas alimentícias divulgadas pelas emissoras, em 336 horas de gravação, $85 \%$ anunciavam produtos contendo açúcares, óleos e gorduras, não sendo observado nenhum comercial abordando o consumo de frutas e hortaliças.

Coll et al.22, em estudo sobre a percepção da influência da mídia sobre o estilo de vida de adolescentes e adultos, observaram que, com relação à alimentação, 89,6\% acreditam que a mídia influencia na mudança de comportamento, sendo que $44,4 \%$ a relataram. Crivelaro ${ }^{23}$ comprova tal afirmação ressaltando que a TV aumenta o risco de obesidade não só por desviar o indivíduo das atividades físicas, mas também por induzir a ingestão de alimentos altamente calóricos.

Ao avaliar os apelos da mídia em comerciais, Domiciano et al. ${ }^{24}$ identificaram uma grande dedicação de marketing por parte das indústrias quanto ao uso de artifícios, principalmente emocionais e afetivos para atrair a atenção dos consumidores, ignorando em quase todos os comerciais o apelo nutricional, comprovando assim o baixo interesse do assunto pelo público. Baseando no raciocínio de Paulo Freire ${ }^{25}$, ao afirmar que "Educação não transforma o mundo. Educação muda pessoas. Pessoas transformam o mundo", pode-se inferir que se o público tivesse melhor educação e, consequentemente, maior interesse pela parte nutricional dos produtos alimentícios, poderia induzir uma mudança de comportamento por parte da divulgação dos produtos e, portanto, maior atenção/dedicação à qualidade nutricional dos alimentos industrializados. Portanto, diante da situação apresentada pelo presente estudo e demais pesquisas com o tema, ressalta-se a necessidade de intervenções que contribuam para a promoção do conhecimento alimentar desse público, contrapondo o exposto pela mídia e dando ao adolescente a ciência e a autonomia necessárias para a adoção de hábitos alimentares saudáveis ao longo da vida, influenciando, assim, positivamente a cultura de uma nação.

Segundo Barlow et al. ${ }^{26}$, intervenções voltadas a crianças e adolescentes, quando bem direcionadas para a promoção de hábitos alimentares saudáveis e acompanhadas do estímulo à prática de exercícios físicos podem desencadear mudanças marcantes e positivas, que podem se estender pela vida adulta, sendo, portanto, a forma mais efetiva para prevenção da obesidade e de doenças crônicas não transmissíveis como diabetes, hipertensão e doenças cardiovasculares. Isso é confirmado por Baranowski et al. ${ }^{6}$ e Shaya et al. ${ }^{9}$, que afirmam que intervenções com crianças e adolescentes são particularmente mais efetivas do que com adultos, sendo as atividades participativas e dinâmicas ferramentas essenciais para promover mudanças efetivas e consistentes. 
O nível de conhecimento dos adolescentes antes e após as intervenções aplicadas no presente estudo estão apresentados na Tabela 2, que mostra as alterações no número de acertos nas respostas do questionário aplicado. A partir destes dados pode-se observar que o grupo B apresentou resultados mais positivos quando comparado ao grupo A, antes da intervenção educativa. Após as intervenções, ambos os grupos apresentaram alterações nos padrões das respostas, indicando aumento do conhecimento em relação às questões avaliadas quando comparado ao momento anterior do processo de educação alimentar e nutricional. No entanto, nos testes efetuados para comparar os resultados encontrados antes e após a realização de ambas as intervenções educativas, observou-se que, estatisticamente, não houve diferença signi- ficativa entre os grupos $(\mathrm{p} \geq 0.05)$. As médias de acertos das questões referentes ao conhecimento dos adolescentes sobre alimentação e nutrição não apresentaram diferença significativa, sendo de aproximadamente 19,45 questões/grupo.

Entretanto, a análise de componentes principais (PCA) mostrou que os resultados obtidos antes e após o processo de EAN apresentaram diferenças entre os grupos, como ilustra a Figura 3. Juntos, os componentes principais explicaram PC1 e PC2 81,47 \% das variações observadas.

Embora os dois grupos não apresentassem distinção de nível de instrução escolar, nota-se na Figura 3 a diferença no grau de conhecimento sobre alimentação e nutrição entre os grupos A e B antes da intervenção educativa. Após a intervenção, os resultados entre os grupos se assemelham,

Tabela 2. Avaliação do grau de conhecimento sobre alimentação e nutrição de adolescentes de uma escola pública do município de Lavras - MG, Brasil.

\begin{tabular}{|c|c|c|c|c|}
\hline \multirow{4}{*}{ Questões } & \multicolumn{4}{|c|}{ Frequência de acertos } \\
\hline & \multicolumn{2}{|c|}{$\begin{array}{c}\text { Turma A } \\
\mathbf{n}=31\end{array}$} & \multicolumn{2}{|c|}{$\begin{array}{c}\text { Turma B } \\
\mathbf{n}=28\end{array}$} \\
\hline & Antes & Depois & Antes & Depois \\
\hline & \multicolumn{4}{|c|}{$\%$} \\
\hline Q2. O que significa Nutrição? & 51.6 & 89.3 & 64.3 & 92.0 \\
\hline Q4. O que um (a) Nutricionista faz? & 77.4 & 82.1 & 82.1 & 80.0 \\
\hline Q5. O que as pessoas comem e bebem podem influenciar na saúde delas? & 96.8 & 92.9 & 100.0 & 100.0 \\
\hline Q6. Quais doenças podem estar relacionadas com a alimentação? & 80.7 & 82.1 & 92.9 & 84.0 \\
\hline Q8. O que significa ter uma alimentação saudável? & 77.4 & 89.3 & 85.7 & 100.0 \\
\hline Q10. O que a Pirâmide Alimentar representa? & 87.1 & 78.6 & 67.9 & 72.0 \\
\hline Q11. Quantas refeições devem ser feitas diariamente? & 61.3 & 64.3 & 60.7 & 76.0 \\
\hline Q12. Quais as refeições mais importantes do dia? & 48.4 & 75.0 & 60.7 & 80.0 \\
\hline $\begin{array}{l}\text { Q13. Uma alimentação saudável é importante para o crescimento e } \\
\text { desenvolvimento? }\end{array}$ & 96.8 & 57.1 & 100.0 & 80.0 \\
\hline Q15. Quais desses itens são considerados nutrientes? & 61.3 & 64.3 & 64.3 & 84.0 \\
\hline Q16. Em quais alimentos encontramos mais carboidrato? & 48.4 & 57.1 & 25.0 & 60.0 \\
\hline Q17. Por que os carboidratos são importantes para o organismo? & 48.4 & 64.3 & 71.4 & 76.0 \\
\hline Q18. Em quais alimentos encontramos mais proteína? & 32.3 & 53.6 & 53.6 & 44.0 \\
\hline Q19. Por que as proteínas são importantes para o organismo? & 45.2 & 75.0 & 35.7 & 72.0 \\
\hline Q20. Em quais alimentos encontramos mais gordura? & 71.0 & 89.3 & 89.3 & 88.0 \\
\hline Q21. Por que as proteínas são importantes para o organismo? & 48.4 & 50.0 & 53.6 & 72.0 \\
\hline Q22. Quais os alimentos fonte de vitamina A, C, D e E? & 64.5 & 57.1 & 50.0 & 60.0 \\
\hline Q23. Quais os alimentos fonte de $\mathrm{Zn}, \mathrm{Se}, \mathrm{Ca}$ e Fe? & 12.9 & 21.4 & 48.4 & 40.0 \\
\hline Q24. Por que as vitaminas e os minerais são importantes para o organismo? & 35.5 & 57.1 & 25.0 & 68.0 \\
\hline Q25. Em quais alimentos encontramos mais fibras? & 74.2 & 64.3 & 71.4 & 44.0 \\
\hline Q26. Por que as fibras são importantes para o organismo? & 51.6 & 78.6 & 21.4 & 92.0 \\
\hline Q27.É importante consumir água várias vezes ao dia? & 100.0 & 100.0 & 96.4 & 96.0 \\
\hline $\begin{array}{l}\text { Q30. Quais informações são importantes de se observar nas embalagens de } \\
\text { alimentos? }\end{array}$ & 35.4 & 67.8 & 35.7 & 48.0 \\
\hline
\end{tabular}


não demonstrando diferença entre os recursos pedagógicos utilizados para a EAN. Contudo, para o mesmo grupo, os resultados posteriores às intervenções educativas diferem daqueles obtidos antes das intervenções, o que assegura que houve alteração no padrão das respostas em ambas as turmas.

Relacionando os resultados encontrados nos testes acima mencionados, pode-se afirmar que, embora não tenha sido encontrada diferença significativa entre as médias de acertos das questões relacionadas ao conhecimento sobre alimentação e nutrição, o padrão das respostas foi alterado positivamente após as intervenções realizadas. A Figura 3 também representa a dispersão das médias de acertos de cada questão.

Segundo esses dados, juntamente com as porcentagens de acerto apresentadas na Tabela 2, antes da intervenção educativa, as questões Q4, Q5, Q6, Q13, Q25 e Q27 apresentavam maior percentual de acertos. Após a intervenção, as questões que tiveram os percentuais mais elevados foram Q2, Q8, Q12, Q15, Q19, Q21, Q24, Q26 e Q30. As demais questões mantiveram seus percentuais de acerto semelhantes, antes e depois do processo de EAN.

Pode-se constatar, portanto, que os dois tipos de intervenção em EAN foram igualmente úteis para promover o conhecimento dos alunos. Embora não considerados estatisticamente significantes pela ANOVA, ambos alteraram a perspectiva de conhecimento sobre alimentação e nutrição entre os adolescentes.

Iuliano et al..$^{27}$, no entanto, demonstraram que as atividades de educação nutricional mais frequentes baseadas na transmissão vertical de informações, na forma de "aula" e "orientações nutricionais informais", que se caracterizam por serem pouco participativas e críticas, como a palestra proposta no presente estudo, apresentamse como estratégias menos eficazes. Ainda segundo os autores, os aspectos lúdicos das atividades quando pouco valorizados, comprometem o envolvimento do educando no processo educativo. Já as atividades participativas como "oficinas de culinária" e "hortas" chamaram maior atenção dos alunos e se mostraram mais eficientes.

Isso foi constatado por Parmer et al. ${ }^{28}$, que demonstraram que a EAN por si só foi ineficiente para promover mudanças nos hábitos alimentares e de comportamento, enquanto que a combinação dessas práticas com atividades direcionadas à implantação de horta escolar promoveu aumento significativo no consumo de frutas, legumes e hortaliças pelas crianças. Muller et al. ${ }^{29}$

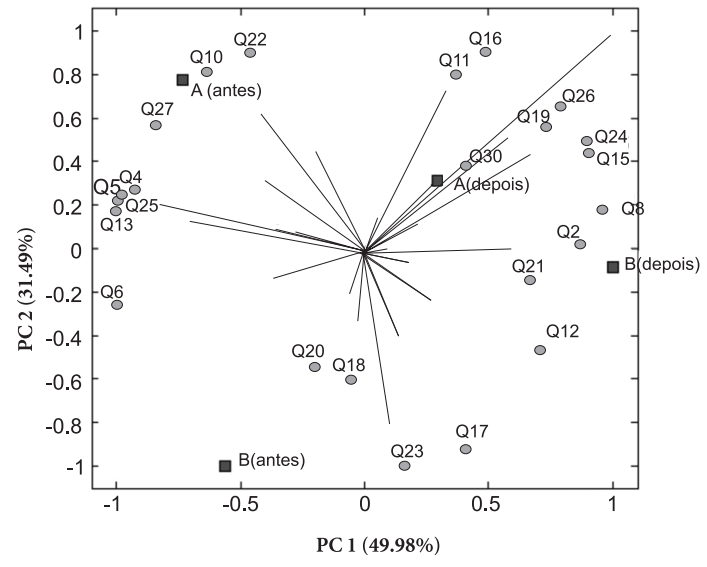

Figura 3. Escores das médias de acertos dos grupos A e $\mathrm{B}$ e pesos das médias de acertos das questões sobre alimentação e nutrição antes e após intervenções educativas.

também reforçam a questão de que é improvável que intervenções isoladas numa única área sejam suficientes para promover mudanças expressivas, alterando positivamente os hábitos alimentares, revertendo, por consequência, o quadro epidemiológico atual.

Portanto, mesmo diante dos resultados positivos obtidos neste estudo para ambos os métodos avaliados, vale ressaltar que as intervenções para serem efetivas e consistentes, devem ir muito além de apenas promover conhecimentos nutricionais. São necessárias ações integradas que visem à saúde das crianças e adolescentes, envolvendo famílias, escolas e comunidades além do apoio e incentivo do governo e universidades para facilitar a promoção de saúde, priorizando a prevenção de doenças e a adoção de hábitos de vida saudáveis.

\section{Conclusão}

No que concerne ao consumo alimentar, encontrou-se um padrão inadequado dos adolescentes em estudo, evidenciando o consumo elevado de alimentos ricos em gorduras e açúcares simples e a baixa ingestão de frutas, verduras e legumes. Já a aplicação do jogo e da palestra como recursos pedagógicos para intervenção educativa foram igualmente úteis para promover o conhecimento dos alunos. 
Diante dos resultados apresentados, demonstra-se a necessidade da implementação de atividades voltadas à educação alimentar e nutricional de adolescentes no ambiente escolar, utilizando diferentes tipos de recursos pedagógicos, de acordo com o objetivo proposto, e integrando escola, família e comunidade na promoção de hábitos alimentares mais saudáveis.

\section{Colaboradores}

TS Pereira participou do planejamento e delineamento do estudo, coleta dos dados, redação da versão preliminar e revisão final do artigo; RC Pereira da interpretação dos dados, redação e revisão final do artigo; MC Angelis-Pereira do planejamento e delineamento do estudo, interpretação dos dados e revisão final do artigo. 


\section{Referências}

1. World Health Organization (WHO). Child and adolescent health and development. Geneva: WHO; 2004.

2. Amaro S, Viggiano A, Di Costanzo A, Madeo I, Viggiano A, Baccari ME, Marchitelli E, Raia M, Viggiano E, Deepak S, Monda M, De Luca B. Kalèdo, a new educational board-game, gives nutritional rudiments and encourages healthy eating in children: a pilot cluster randomized trial. Eur J Pediatr 2005; 165(9):630-635.

3. Yang YTC, Wang CJ, Tsai MF, Wang JS. Technology-enhanced game-based team learning for improving intake of food groups and nutritional elements. Comput Educ 2015; 88:143-159.

4. Elliot P, Daniels S. Pestalozzi, Fellenberg and British nineteenth-century geographical education. J Hist Geogr 2006; 32(4):752-774.

5. Silva ECR, Fonseca AB. Abordagens pedagógicas em educação alimentar e nutricional em escolas no Brasil. In: Encontro Nacional de Pesquisa em Educação em Ciência, 2009; Florianópolis.

6. Baranowski T, Baranowski J, Cullen KW, Marsh T, Islam N, Zakeri I, Honess-Morreale L, deMoor C. Squire's Quest! Dietary outcome evaluation of a multimedia game. Am J Prev Med 2003; 24(1):52-61.

7. Waters E, Silva-Sanigorski A de, Burford BJ, Brown T, Campbell KJ, Gao Y, Armstrong R, Presser L, Summerbell $\mathrm{CD}$. Interventions for preventing obesity in children. Cochrane Database Syst Rev 2011; 12:CD001871

8. Contento IR, Randell JS, Basch C. Review and analysis of evaluation measures used in nutrition education intervention research. J Nutr Educ Behav 2002; 34(1):225.

9. Shaya FT, Flores D, Gbarayor CM, Wang J. Schoolbased obesity interventions: a literature review. J Sch Health 2008; 78(4):189-196.

10. Nunes CA. Chemoface [programa de computador]. Versão 1.5. Lavras: UFLA; 2012.

11. Philippi ST, Latterza AR, Cruz ATR, Ribeiro LC. Pirâmide alimentar adaptada: guia para escolha dos alimentos. Rev Nutr 1999; 12(1):65-80.

12. Brasil. Ministério da Saúde (MS). Guia alimentar para a população brasileira: promovendo a alimentação saudável. Brasília: MS; 2005.

13. Carmo MB, Toral N, Silva MV, Slater B. Consumo de doces, refrigerantes e bebidas com adição de açúcar entre adolescentes da rede pública de ensino de Piracicaba, São Paulo. Rev Bras Epidemiol 2006; 9(1):121-130.

14. Salles-Costa R. Avaliação alimentar em Duque de Caxias - RJ. Estudos Avançados 2007; 21(60):135-142.

15. Toral N, Slater B, Silva MV. Consumo alimentar e excesso de peso de adolescentes de Piracicaba, São Paulo. Rev Nutr 2007; 20(5):449-459.

16. Enes CC, Pegolo GE, Silva MV. Hábitos alimentares de adolescentes residentes em áreas rurais da cidade de Piedade, São Paulo. Nutrire 2008; 33(2):99-110.

17. Mendes KL, Catão LP. Avaliação do consumo de frutas, legumes e verduras por adolescentes de Formiga - MG e sua relação com fatores socioeconômicos. Alim Nutr 2010; 21(2):291-296.
18. Monticelli FDB, Souza JMP, Souza SB. Consumo de frutas, legumes e verduras por escolares adolescentes. J Hum Growth Dev, 2013; 23(3):331-337.

19. Freitas LKP, Cunha JAT, Knackfuss MI, Medeiros HJ. Obesidade em adolescentes e as políticas públicas de nutrição. Cien Saude Colet 2014; 19(6):1755-1762.

20. Wong LLR, Carvalho JA. O rápido processo de envelhecimento populacional do Brasil: sérios desafios para as políticas públicas. Rev Bras Estud de Popul 2006; 23(1):5-26.

21. Santos CC, Stuchi RAG, Arreguy-Sena C, Pinto NAVD. A influência da televisão nos hábitos, costumes e comportamento alimentar. Cogitare Enfermagem 2012; 17(1):65-71

22. Coll C, Bustos A, Engel A. As comunidades virtuais de aprendizagem. In: Coll C, Monereo C, organizadores. Psicologia da Educação Virtual: aprender e ensinar com as tecnologias de informação e da comunicação. Porto Alegre: Artmed; 2010. p. 268-286.

23. Crivelaro LP. A publicidade na TV e sua influência na obesidade infantil. UNIrevista 2006; 1(3):1-7.

24. Domiciano CG, Coelho LR, Pereira JAR, de Angelis-Pereira MC. Estratégias da mídia e os apelos comerciais para promoção dos produtos alimentícios. Rev Ciênc Saúde 2014; 4(1):1-7.

25. Freire P. Educação como prática da liberdade. Rio de Janeiro: Paz e Terra; 1999.

26. Barlow SE, Trowbridge FL, Klish WJ, Dietz WH. Treatment of child and adolescent obesity: reports from pediatricians, pediatric nurse practitioners, and registered dietitians. Pediatrics 2002; 110(1 Pt 2):229-235.

27. Iuliano BA, Mancuso AMC, Gambardella AMD. Educação nutricional em escolas de ensino fundamental do município de Guarulhos-SP. O Mundo da Saúde 2009; 33(3):264-272.

28. Parmer SM, Salisbury-Glennon J, Shannon D, Struempler B. School gardens: an experiential learning approach for a nutrition education program to increase fruit and vegetable knowledge, preference, and consumption among second-grade students. J Nutr Educ Behav 2009; 41(3):212-217.

29. Müller MJ, Mast M, Asbeck I, Langnäse K, Grund A. Preventions of obesity - it is possible? Obes Rev 2001; 2(1):15-28.

Artigo apresentado em 02/09/2015

Aprovado em 17/12/2015

Versão final apresentada em 19/12/2015 
